\title{
On exact proper time Wilsonian RG flows
}

\author{
Alfio Bonanno ${ }^{1,2, a}$, Stefan Lippoldt ${ }^{3, b}$, Roberto Percacci ${ }^{4,5, c}$, Gian Paolo Vacca ${ }^{6, \mathrm{~d}}$ \\ ${ }^{1}$ INAF, Osservatorio Astrofisico di Catania, via S.Sofia 78, 95123 Catania, Italy \\ 2 INFN, Sezione di Catania, via S. Sofia 64, 95123 Catania, Italy \\ ${ }^{3}$ Institut für Theoretische Physik, Universität Heidelberg, Philosophenweg 16, 69120 Heidelberg, Germany \\ ${ }^{4}$ SISSA, via Bonomea 265, 34136 Trieste, Italy \\ 5 INFN, Sezione di Trieste, Trieste, Italy \\ ${ }^{6}$ INFN, Sezione di Bologna, via Irnerio 46, 40126 Bologna, Italy
}

Received: 2 February 2020 / Accepted: 1 March 2020 / Published online: 18 March 2020

(C) The Author(s) 2020

\begin{abstract}
We discuss the possibility to define exact RG equations for a UV regulated Wilsonian action based on a proper time (PT) regulator function. We start from a functional mapping which shows how each particular flow equation (and RG scheme) is associated to infinitely many scale dependent field redefinitions, which are related to specific coarse-graining procedures. On specializing to a sub-family of one parameter PT regulators we briefly analyze few results for the Ising Universality class in three dimensions, obtained within a second order truncation in the derivative expansion of the Wilsonian action.
\end{abstract}

\section{Introduction}

When a generating functional in a quantum field theory is defined by means of a functional integral with a built-in cutoff, its derivative with respect to the cutoff gives rise to a Renormalization Group (RG) flow. The original idea is due to Wilson [1], who defined an action $S_{\Lambda}$ depending on an UV cutoff $\Lambda$ in such a way that when $S_{\Lambda}$ is used in a functional integral with a cutoff $\Lambda$, it gives the same partition function independently on the choice of $\Lambda$. In other words, the physical predictions at low energy are unchanged:

$Z=\int[d \varphi]_{\Lambda} e^{-S_{\Lambda}[\varphi]}, \Lambda \frac{\mathrm{d}}{\mathrm{d} \Lambda} Z=0$.

The subscript $\Lambda$ in the measure means that momentum integrals are to be cut off in the UV at $\Lambda$. There is no unique way to define the cutoff, but all these Wilsonian RG flows

\footnotetext{
a e-mail: alfio.bonanno@inaf.it

b e-mail: s.lippoldt@thphys.uni-heidelberg.de

c e-mail: percacci@sissa.it (corresponding author)

de-mail: vacca@bo.infn.it
}

can be shown to satisfy some kind of differential equation, usually called an Exact RG Equation (ERGE). One of the oldest forms of the ERGE is due to Wegner and Houghton [2]. Better-known in the context of particle physics is the Polchinski equation [3], giving the flow of the interaction part of the action.

A different class of equations arise when we consider the generating functional of 1-particle irreducible correlators, the Effective Action (EA) $\Gamma$. In this case one introduces in the functional integral an IR cutoff, usually called $k$, and the functional $\Gamma_{k}$ is called the Effective Average Action (EAA) [4]. It satisfies an exact RG equation known as the Wetterich equation [5-8]. Employing various approximation schemes, these exact equations can be used to derive many results in condensed matter, statistical and particle physics. For reviews, see [9-13]. For applications to gauge theories see [14-16].

At one loop, in the Schwinger proper time (PT) representation of the effective action, one can introduce the cutoff (either UV or IR or both) in the integral over the proper time, rather than the integral over momenta $[17,18]$. The resulting "proper time RG equation" for the EAA is known not to be exact [19-21], but nevertheless has been applied to various problems from statistical physics $[22,23]$ to gravity [24], and gives comparable results to the various forms of the ERGE. This is generally attributed to the fact that the one loop approximation becomes exact when one considers integration over infinitesimal momentum shells [2]. Recently, a PT-like equation for the Wilsonian action, rather than the EAA, has been introduced in [25]:

$$
\Lambda \frac{d S_{\Lambda}[\varphi]}{d \Lambda}=\operatorname{Tr} e^{-S_{\Lambda}^{(2)}[\varphi] / \Lambda^{2}}
$$

It has been applied to issues of quantum gravity. Unlike previous PTRG equations, this one is claimed to be exact. We stress that this would not be in contrast with the previous statement 
for the 1PI functional (EAA) because now the equation holds for a Wilsonian action $S_{\Lambda}$, which has to be used in the functional integral of the low momentum modes. In this paper we will examine by different means a more general family of equations which can characterize the RG flow of the Wilsonian action $S_{\Lambda}$ and, as a simple quantitative test, apply it to derive the scaling exponents of the Ising universality class.

\section{Wilsonian PTRG equations}

Consider a general family of PT flows

$\Lambda \frac{d S_{\Lambda}[\varphi]}{d \Lambda}=\frac{1}{2} \operatorname{Tr} \int_{0}^{\infty} \frac{d s}{s} r_{\Lambda}(s) e^{-s S_{\Lambda}^{(2)}[\varphi]}$.

Here $s$ is the Schwinger proper time,

$r_{\Lambda}(s)=\Lambda \frac{d \rho_{k, \Lambda}}{d \Lambda}$

and $\rho_{k, \Lambda}(s)$ is a suitably normalized regulator function depending on an IR scale $k$ and an UV scale $\Lambda$. It goes to zero for $s<1 / \Lambda^{2}$ (UV regularization) and $s>1 / k^{2}$ (IR regularization), and is roughly equal to one in between. Choosing the regulator function

$\rho_{k, \Lambda}(s)=\theta\left(s-1 / \Lambda^{2}\right)-\theta\left(s-1 / k^{2}\right)$,

one obtains de Alwis' equation (2). We will now discuss other choices of regulator. Let us consider the following 1parameter family of regulators $(m \geq 0)$,

$\rho_{k, \Lambda}(s ; m)=\frac{\Gamma\left(m, m s k^{2}\right)-\Gamma\left(m, m s \Lambda^{2}\right)}{\Gamma(m)}$.

Using the properties of the incomplete Gamma function we obtain

$r_{\Lambda}(s ; m)=\frac{2}{\Gamma(m)}\left(m s \Lambda^{2}\right)^{m} e^{-m s \Lambda^{2}}$.

Note that this object is actually dependent on the UV cutoff $\Lambda$ only, justifying the notation (4). We shall call this the "A-scheme". Inserting this choice of regulator in (3) and performing the Mellin transform we get the following RG equation for the Wilsonian action $S_{\Lambda}$,

$\Lambda \frac{d}{d \Lambda} S_{\Lambda}[\varphi]=\operatorname{Tr}\left(\frac{m \Lambda^{2}}{S_{\Lambda}^{(2)}[\varphi]+m \Lambda^{2}}\right)^{m}$.

Note that for $m=1$ this looks like the Wetterich Equation with a massive regulator (which can define a flow for the IR regulated effective average action in low dimensionality). In the LPA approximation, the specific value $m=d / 2+1$ gives essentially the same flow for the potential as the Wetterich equation with an optimized regulator. One should not be misled by these analogies, since $S_{\Lambda}$ has to be interpreted only as a Wilsonian action (to appear inside a functional integral) and not as an IR-regulated generator of the 1PI correlators [26].

Note also that for $m \rightarrow \infty$ the scale derivative of $\rho_{k, \Lambda}(s)$ becomes a Dirac delta distribution, or equivalently, one has in (8) the representation of an exponential, so the flow equation reduces again to the one given in Eq. (2).

The cutoffs (5) only depend on $s, k$ and $\Lambda$. It is also possible to introduce in the PT regulator some field dependence. This is related to the idea of "spectral adjustment". Let us start by considering the derivative expansion of a generic Wilsonian action $S_{\Lambda}(\varphi)$, with $S_{\Lambda}^{(2)}(\varphi)=Z_{\Lambda}(\varphi)(-\square)+\cdots$. Here $\varphi$ has to be slowly varying on length scales of order $p^{-1}$, where $p$ are the momenta appearing in $-\square$. In Eq. (3), the regulator $\rho(s)$ suppresses the part of the integral with $s<1 / \Lambda^{2}$, while the exponential suppresses modes with $Z_{\Lambda} p^{2}>1 / s$. Altogether the suppression occurs for $Z_{\Lambda} p^{2}>\Lambda^{2}$. We see that if $Z_{\Lambda}$ is sizable, the cutoff is not directly related to the spacetime scale of the fluctuation: this scale is modulated by a flowing field-dependent dynamical factor $Z_{\Lambda}$. Let us consider instead a more "spectrally adjusted" scheme obtained using a proper time regulator $\rho\left(Z_{\Lambda} s\right)$. In this case the regulator suppresses the part of the integral with $s<\frac{1}{\Lambda^{2} Z_{\Lambda}}$, which corresponds to a suppression of the modes with $p^{2}>\Lambda^{2}$. This is closer to the original intent of cutting off the modes at the sliding scale $\Lambda$. One observes that it is in the spectrally adjusted scheme that the two steps in the renormalization procedure, namely (1) coarse-graining and (2) rescaling, are tuned to each other as desired for a comparison along the flow.

If we "spectrally adjust" in this fashion the cutoff (6)

$\rho_{k, \Lambda}(s ; m)=\frac{\Gamma\left(m, m s Z_{k}(\phi) k^{2}\right)-\Gamma\left(m, m s Z_{\Lambda}(\phi) \Lambda^{2}\right)}{\Gamma(m)}$.

we obtain

$\begin{aligned} r_{\Lambda}(s ; m)= & \frac{2}{\Gamma(m)}\left(1+\frac{1}{2} \Lambda \frac{d}{d \Lambda} \log Z_{\Lambda}(\phi)\right) \\ & \times\left(m s Z_{\Lambda}(\phi) \Lambda^{2}\right)^{m} e^{-m s Z_{\Lambda}(\phi) \Lambda^{2}}\end{aligned}$

We call this the "B scheme". Then inserting in (3) we arrive at the RG equation

$$
\begin{aligned}
\Lambda \frac{d}{d \Lambda} S_{\Lambda}[\varphi] & =\operatorname{Tr}\left[\left(1+\frac{1}{2} \Lambda \frac{d}{d \Lambda} \log Z_{\Lambda}\right)\right. \\
& \left.\times\left(\frac{m \Lambda^{2} Z_{\Lambda}(\phi)}{S_{\Lambda}^{(2)}[\varphi]+m \Lambda^{2} Z_{\Lambda}(\phi)}\right)^{m}\right] .
\end{aligned}
$$

In the limit $m \rightarrow \infty$ one gets

$\Lambda \frac{d}{d \Lambda} S_{\Lambda}[\varphi]=\operatorname{Tr}\left[\left(1+\frac{1}{2} \Lambda \frac{d}{d \Lambda} \log Z_{\Lambda}(\phi)\right) e^{-\frac{S_{\Lambda}^{(2)}[\varphi]}{\Lambda^{2} Z_{\Lambda}(\phi)}}\right]$. 
In the preceding discussion we have assumed that the "spectral adjustment" is introduced at the level of the cutoff function $\rho_{k, \Lambda}$. In principle, it could also be carried out at the level of the infinitesimal RG transformation (4), or (7), namely

$r_{\Lambda}(s ; m)=\frac{2}{\Gamma(m)}\left(m s Z_{\Lambda}(\phi) \Lambda^{2}\right)^{m} e^{-m s \Lambda^{2}}$.

This then leads to an RG equation that is similar to (12), but without the term $\Lambda \frac{d}{d \Lambda} \log Z_{\Lambda}(\phi)$. We will refer to it as the "C scheme".

\section{Relation to formal coarse-graining schemes}

The main goal of this Section is to investigate in a formal way if the family of flow equations Eq. (3), can be interpreted as Wilsonian flows. In general terms the coarse-graining procedure for the Wilsonian action can be defined as

$\Lambda \frac{d}{d \Lambda} e^{-S_{\Lambda}[\varphi]}=\int d x \frac{\delta}{\delta \varphi(x)}\left(\psi_{x}^{\Lambda}[\varphi] e^{-S_{\Lambda}[\varphi]}\right)$,

for some $\psi_{x}^{\Lambda}[\varphi]$. This equation implies that the partition function $Z=\int[d \varphi] e^{-S_{\Lambda}[\varphi]}$ is manifestly independent of $\Lambda$. The specific form of the quantity in brackets leads to the following general form for Wilsonian flow:

$\Lambda \frac{d}{d \Lambda} S_{\Lambda}[\varphi]=\int d x\left(\frac{\delta S_{\Lambda}[\varphi]}{\delta \varphi(x)} \psi_{x}^{\Lambda}[\varphi]-\frac{\delta \psi_{x}^{\Lambda}[\varphi]}{\delta \varphi(x)}\right)$.

We also recall that this general Wilsonian flow is associated to an infinitesimal field redefinition $\varphi(x) \rightarrow \varphi^{\prime}(x)=\varphi(x)-$ $\frac{\delta \Lambda}{\Lambda} \psi_{x}^{\Lambda}[\varphi]$. Indeed starting from a given coarse-graining map $\varphi \rightarrow b_{\Lambda}(\varphi)$, transforming the field $\varphi_{0}$ at the scale $\Lambda_{0}$ into the field $\varphi$ at the scale $\Lambda<\Lambda_{0}$, one can derive the following relation $[13,27]$

$$
\begin{aligned}
\psi_{x}^{\Lambda}[\varphi] & =e^{S_{\Lambda}[\varphi]} \int\left[d \varphi_{0}\right] \delta\left(\varphi-b_{\Lambda}\left[\varphi_{0}\right]\right) \\
& \times \Lambda \frac{d b_{\Lambda}\left[\varphi_{0}\right](x)}{d \Lambda} e^{-S_{\Lambda_{0}}\left[\varphi_{0}\right]} .
\end{aligned}
$$

It is then natural to ask whether the general flow Eq. (15) can be reduced to the PT flow in Eq. (3), and if so, what conditions should $\psi_{x}^{\Lambda}[\varphi]$ satisfy.

Let us first recall what happens in the case of the Wilsonian flow considered by Polchinski [3]. In this case one can directly guess the form of $\psi_{x}^{\Lambda}[\varphi]$ to be plugged in the general formula (15):

$\psi_{x}^{\Lambda}[\varphi]=\frac{1}{2} \int d z \dot{\Delta}_{x z} \frac{\delta \Sigma_{\Lambda}[\varphi]}{\delta \varphi(z)}$,

where $\Delta$ is a suitably regulated propagator with a dot standing for the derivative w.r.t. $\log \Lambda$ and $\Sigma_{\Lambda}[\varphi]$ is given by

$$
\Sigma_{\Lambda}[\varphi]=-\frac{1}{2} \int d x \varphi(x)\left(-\square_{x}\right) \varphi(x)+S_{\Lambda}^{I}[\varphi],
$$

$$
S_{\Lambda}[\varphi]=\frac{1}{2} \int d x \varphi(x)\left(-\square_{x}\right) \varphi(x)+S_{\Lambda}^{I}[\varphi] .
$$

This choice leads to the desired flow equation

$$
\begin{aligned}
\Lambda \frac{d}{d \Lambda} S_{\Lambda}^{I}[\varphi]= & \frac{1}{2} \int d x d y \dot{\Delta}_{x y}\left[\frac{\delta S_{\Lambda}^{I}[\varphi]}{\delta \varphi(y)} \frac{\delta S_{\Lambda}^{I}[\varphi]}{\delta \varphi(x)}\right. \\
& \left.-\frac{\delta^{2} S_{\Lambda}^{I}[\varphi]}{\delta \varphi(y) \delta \varphi(x)}\right] .
\end{aligned}
$$

The same RG flow equation can be actually obtained also by other choices of $\psi_{x}^{\Lambda}[\varphi]$, belonging to an infinite family obtained adding to the expression in Eq. (17) a term $w_{x}[\varphi] e^{s_{\Lambda}[\varphi]}$, such that $\int d x \frac{\delta}{\delta \varphi(x)} w_{x}[\varphi]=0$. They correspond to different (but equivalent, in generating the flow) implementations of the coarse-graining procedure.

The Polchiski Wilsonian effective action $S_{\Lambda}$, which satisfies the above flow equation, gets, in general, both 1PI (the second term) and 1PR (the first term) contributions. It is known in this case that only in the sharp cutoff limit, if the momenta flowing into a vertex of a 1PR term have sum below the UV cutoff $\Lambda$, then such contributions are absent [6]. Similar considerations are valid for the Wegner-Houghton Wilsonian action. Moreover the Polchinski action has a simple relation with the regulated generator of the connected Green's functions and with the effective average action [6].

Going back to the goal of this section, in order to interpret in the Wilsonian sense the general PT flow Eq. (3), we would have to rewrite it in the form given in the Eqs. (15) or (14). Thus we must look for a solution of the following functional equation

$$
\begin{aligned}
& \int d x \frac{\delta}{\delta \varphi(x)}\left(\psi_{x}^{\Lambda}[\varphi] e^{-S_{\Lambda}[\varphi]}\right) \\
& =-e^{-S_{\Lambda}[\varphi]} \frac{1}{2} \operatorname{tr} \int_{0}^{\infty} \frac{d s}{s}\left[r_{\Lambda}(s) e^{-s S_{\Lambda}^{(2)}[\varphi]}\right] .
\end{aligned}
$$

Before moving to this task let us make a comment. The existence of a solution would make it possible to interpret this particular PT regulated action $S_{\Lambda}$, as a Wilsonian action, i.e. an action which, inserted in a functional integral, not only generates the partition function $Z$, but also all the possible correlators (connected and not connected) with momenta below the scale $\Lambda$, i.e.

$$
\begin{aligned}
& \left\langle O_{1}\left(x_{1}\right) \cdots O_{n}\left(x_{n}\right)\right\rangle \\
& =\frac{1}{Z} \int[d \varphi]_{\Lambda} e^{-S_{\Lambda}[\varphi]} O_{1}\left(x_{1}\right) \cdots O_{n}\left(x_{n}\right) .
\end{aligned}
$$

From the structure of the flow equation one notes that in general, contrary to the Polchinski action, this action gets along the flow contributions which results into 1PI non local vertices. We stress that the relation between the PT regulated $S_{\Lambda}$ and the effective action $\Gamma$ (eventually IR regulated) is not trivial and certainly not so simple as for the Polchinski Wilsonian action. 


\subsection{Construction of a general solution}

Let us then ask the following question: given a certain Wilsonian $\mathrm{RG}$ flow is it possible to reconstruct the associated coarse-graining procedure which may be encoded in the infinitesimal generator $\psi_{x}^{\Lambda}[\varphi]$ ? We have already seen for the specific case of a Polchinski RG flow that this procedure is not unique. From the definition recalled above it is clear that such a construction requires knowledge of the Wilsonian action $S_{\Lambda}[\varphi]$ satisfying the RG flow equation, making this problem hard to solve in practice. Nevertheless it is important to know if at least the solution of this problem can exist.

The left hand side of this equation can be seen as a divergence of a vector field belonging to an infinite dimensional vector space, i.e., has the structure

$\int d x \frac{\delta}{\delta \varphi(x)} u_{x}[\varphi]=f[\varphi]$

Keeping in mind that the quadratic kinetic part of the Wilsonian action is defined by a UV regulated Laplacian $(\square)_{\Lambda}$, it is convenient to define a slightly different object, which already simplifies the analysis for the free theory case as discussed in the next subsection, ${ }^{1}$

$u_{x}[\varphi]=G_{x y} v_{y}[\varphi]$

where we have introduced the space-time 'regulated' Green's function $G_{x y}$ satisfying

$\left(-\square_{x}\right)_{\Lambda} G_{x y}=\delta(y)$.

One can assume that as in any finite dimensional vector space, the vector field $v_{x}[\varphi]$ can be decomposed as a sum of a "gradient" part and a divergenceless part as follows

$$
\begin{aligned}
v_{x}[\varphi] & =\frac{\delta}{\delta \varphi(x)} h[\varphi]+\left(-\square_{x}\right)_{\Lambda} w_{x}[\varphi], \\
& \times \int d x \frac{\delta}{\delta \varphi(x)} w_{x}[\varphi]=0 .
\end{aligned}
$$

Then a particular solution of Eq. (22) using Eq. (25) can be obtained solving an infinite dimensional Poisson-like equation

$$
\int d x d y \frac{\delta}{\delta \varphi(x)} G_{x y} \frac{\delta}{\delta \varphi(y)} h[\varphi]=f[\varphi],
$$

where the metric in field space is field independent (flat geometry in field space) even if has a non trivial space-time dependence. Since the determinant of the metric is field independent one can see that the operator in field space above is really a covariant Laplacian. We expect that this generalized "elliptic" second order linear differential problem can have solutions, unique at least from the physical point of view when suitable boundary conditions are imposed.

\footnotetext{
1 We thank Tim Morris for this suggestion.
}

Let us write a functional Fourier transform for the scalar $h$ introducing a source $J$

$\tilde{h}[J]=\int[d \varphi] e^{-i \varphi \cdot J} h[\varphi]$

and similarly for the right hand side of Eq. (26), i.e. for $f[\varphi]$. Then one can formally rewrite this generalized Poisson equation as

$-(J \cdot G \cdot J) \tilde{h}[J]=\tilde{f}[J]$,

where $J \cdot G \cdot J=\int d x d y J(x) G_{x y} J(y)$, and derive its solution

$h[\varphi]=-\int[d J] e^{i \varphi \cdot J} \frac{1}{J \cdot G \cdot J} \tilde{f}[J]$.

Using this setup for our original problem we can then formally write

$\psi_{x}^{\Lambda}[\varphi]=e^{S_{\Lambda}[\varphi]}\left(\int[d J] e^{i \varphi \cdot J} \frac{-i J(x)}{J \cdot G \cdot J} \tilde{f}[J]+w_{x}[\varphi]\right)$,

where $w_{x}[\varphi]$ is an arbitrary divergenceless vector field according to Eq. (25), which again can be chosen to improve the behavior of the solution. Therefore we can see that formally, given a Wilsonian flow, one can construct a coarsegraining procedure which generates it. Moreover the coarsegraining procedure is not unique, indeed there are infinitely many. One can take eventually advantage of this freedom to make the most sensible choice of coarse-graining from the physical point of view.

One can even try to formalize this picture considering the space of Wilsonian actions $\mathcal{S}$ and the space of Wilsonian RG flows $\mathcal{V}$, where each point $p \in \mathcal{V}$ is the vector field functional of the flow, e.g., associated to Eq. (15). Then one can consider a fiber bundle space $\mathcal{F}$ such that, to each point $p$ belonging to its base $\mathcal{V}$ is associated a fiber related to the coarse-graining generator $\psi_{x}^{\Lambda}$. Like in a gauge theory an infinite set of $\psi_{x}^{\Lambda}$ is associated to the same vector field $p$ generating the Wilsonian RG flow.

In summary at least formal solutions of Eq. (20) can be constructed. This is true also in the spectrally adjusted cases named B and C in the end of Sect. 2, which are the ones which make it possible to interpret a proper time coarse-graining in terms of a momentum coarse-graining in the derivative expansion. This seems to confirm that the general PT regulated flow Eq. (3) can represent a Wilsonian RG flow.

\subsection{The free theory case}

Given that a formal solution can be constructed, one may ask which kind of requirements should be further imposed on the field redefinitions by the Wilsonian coarse graining procedure from a reasonable physical point of view. Clearly 
one would like to avoid possible pathological definitions. This question can be posed in general and in particular for the specific case of a proper time flow. First one can notice that even in the Polchinski flow for a free theory, according to Eqs. (17) and (18), there is some degree of non locality in the redefinition of the fields, which depends on the regulator properties encoded in $\Delta$. Further non local behavior, at least in the IR regime, is expected in presence of new degrees of freedom in the spectrum of the theory, such as bound states.

We shall then investigate what happens for the case of a proper-time flow, but which could hold also for more general class of flows. Let us then investigate in detail the case of a free quadratic action for the case of a generic cutoff

$$
\begin{aligned}
& S[\varphi]=\frac{1}{2} \varphi \cdot(-\square)_{\Lambda} \cdot \varphi, \\
& f[\varphi]=-\frac{1}{2} e^{-S_{\Lambda}[\varphi]} \operatorname{Tr} \int_{0}^{\infty} \frac{d s}{s}\left[r_{\Lambda}(s) e^{-s(-\square)_{\Lambda}}\right]
\end{aligned}
$$

so that one can write

$$
\begin{aligned}
\tilde{f}[J]= & -\frac{1}{2} \operatorname{Tr} \int_{0}^{\infty} \frac{d s}{s}\left[r_{\Lambda}(s) e^{-s(-\square)_{\Lambda}}\right] \\
& \times(\operatorname{Det} G)^{\frac{1}{2}} e^{-\frac{1}{2} J \cdot G \cdot J} .
\end{aligned}
$$

Using Eq. (30) we can then write the solution for the "potential" $h$ as

$$
\begin{aligned}
h[\varphi]= & \frac{1}{2} \operatorname{Tr} \int_{0}^{\infty} \frac{d s}{s}\left[r_{\Lambda}(s) e^{-s(-\square)_{\Lambda}}\right] \int[d J] e^{i \varphi \cdot J} \\
& \times \frac{(\operatorname{Det} G)^{\frac{1}{2}}}{J \cdot G \cdot J} e^{-\frac{1}{2} J \cdot G \cdot J} \\
= & \mathcal{N} \int[d \tilde{J}] e^{i \tilde{J} \cdot G^{-\frac{1}{2}} \varphi} \frac{1}{\tilde{J} \cdot \tilde{J}} e^{-\frac{1}{2} \tilde{J} \cdot \tilde{J}},
\end{aligned}
$$

where $\tilde{J}=G^{\frac{1}{2}} J$ and we have defined the normalization factor

$\mathcal{N}=\frac{1}{2} \operatorname{Tr} \int_{0}^{\infty} \frac{d s}{s}\left[r_{\Lambda}(s) e^{-s(-\square)_{\Lambda}}\right]$.

One has to keep in mind that a functional derivative must be taken to construct $v_{x}$ and the infinitesimal Wilsonian field redefinition $\Psi_{x}^{\Lambda}$ as well, so that in the integral in Eq. (33) the singular region in the origin (in $\tilde{J}$ space) is harmless and one could replace $e^{i \tilde{J} \cdot G^{-\frac{1}{2}} \varphi} \rightarrow\left(e^{i \tilde{J} \cdot G^{-\frac{1}{2}} \varphi}-1-i \tilde{J} \cdot G^{-\frac{1}{2}} \varphi\right)$, given that non zero contributions in the integral in $\tilde{J}$ comes from even integrands. One can then perform the Fourier transform.

We follow here an alternative path in the computation by introducing a parameter $a$ to be set to $\frac{1}{2}$ at the end in the previous formal solution for $h[\varphi]$ :

$I_{a}[\varphi]=\mathcal{N} \int[d \tilde{J}] e^{i \tilde{J} \cdot G^{-\frac{1}{2}} \varphi} \frac{1}{\tilde{J} \cdot \tilde{J}} e^{-a \tilde{J} \cdot \tilde{J}}$, and, after taking a derivative w.r.t. $a$, perform the $\tilde{J}$ functional integration

$$
\begin{aligned}
-\frac{d}{d a} I_{a}[\varphi] & =\mathcal{N} \int[d \tilde{J}] e^{i \tilde{J} \cdot G^{-\frac{1}{2}} \varphi} e^{-a \tilde{J} \cdot \tilde{J}} \\
& =\mathcal{N} \frac{\sqrt{\pi}}{\sqrt{a}} e^{-\frac{\varphi \cdot(-\square)_{\Lambda} \cdot \varphi}{4 a}} .
\end{aligned}
$$

Therefore $I_{a}$ can be thought of as a function of $S_{\Lambda}[\varphi]=$ $\frac{1}{2} \varphi \cdot(-\square)_{\Lambda} \cdot \varphi$. Re-integrating back in $a$ one finds

$$
\begin{aligned}
I_{a}[\varphi]= & -\mathcal{N}\left[2 e^{-\frac{\varphi \cdot(-\square)_{\Lambda} \cdot \varphi}{4 a}} \sqrt{\pi a}+\pi \sqrt{\varphi \cdot(-\square)_{\Lambda} \cdot \varphi}\right. \\
& \left.\operatorname{erf}\left(\sqrt{\frac{\varphi \cdot(-\square)_{\Lambda} \cdot \varphi}{4 a}}\right)+c[\varphi]\right],
\end{aligned}
$$

where the last term is an $a$-independent functional. From a direct integration it turns out to be a simple constant and can be discarded. Setting $a=1 / 2$ and ignoring also $w_{x}[\varphi]$ in Eq. (25) one gets

$$
\begin{aligned}
\psi_{x}[\varphi]= & e^{-\frac{1}{2} \varphi \cdot(-\square)_{\Lambda} \cdot \varphi} G_{x y} \frac{\delta h[\varphi]}{\delta \varphi(y)} \\
= & -\mathcal{N} e^{-\frac{1}{2} \varphi \cdot(-\square)_{\Lambda} \cdot \varphi} \frac{\pi}{\sqrt{\varphi \cdot(-\square)_{\Lambda} \cdot \varphi}} \\
& \times \operatorname{erf}\left(\sqrt{\frac{\varphi \cdot(-\square)_{\Lambda} \cdot \varphi}{2}}\right) \varphi_{x} \\
= & -\mathcal{N} \sqrt{2 \pi}\left(1-\frac{2}{3} \varphi \cdot(-\square)_{\Lambda} \cdot \varphi\right. \\
& +\frac{7}{30}\left(\varphi \cdot(-\square)_{\Lambda} \cdot \varphi\right)^{2} \\
& \left.-\frac{2}{35}\left(\varphi \cdot(-\square)_{\Lambda} \cdot \varphi\right)^{3}+\cdots\right) \varphi_{x} .
\end{aligned}
$$

We note that in this free theory the coarse-graning is, as said, a function of the free Wilsonian action, and therefore non local, however this non locality is harmless in this case.

More generally, in presence of interactions, any analysis is extremely complicated and it could be carried on only with drastic simplifications. A fortiori in this case we should not expect in general quasi locality in the action, e.g. in strongly interacting theories where bound states appear in the spectrum. In such cases the flow equation is also bound to be nonlocal. We note, however, that infinitely many coarse-graining schemes give the same flow and that the freedom to introduce the divergenceless vector in field space, as defined in Eq. (25) may be helpful to eliminate some pathological nonlocalities. Similar considerations can be applied in a quasi local regime, i.e. when the action can be written as a power series in derivatives. 


\section{Working example: the Wilson-Fisher fixed point}

It is interesting to apply this formalism to the determination of critical exponents for the Wilson-Fisher fixed point. Let us therefore consider the following euclidean action, which represents the leading term in a derivative expansion:

$S_{\Lambda}[\phi]=\int d^{d} x\left(\frac{1}{2} Z_{\Lambda}(\phi) \partial_{\mu} \phi \partial^{\mu} \phi+V_{\Lambda}(\phi)\right)$,

We will study the flow of this action with a modified type-A regulator. In order to take into account the anomalous dimensions, we shall assume that the field does not have the standard mass dimension $d / 2-1$ but rather $d / 2-1+\eta / 2$. Then the prefactor $Z_{\Lambda}(\phi)$ has dimension $-\eta$. Accordingly, we modify the cutoffs of Eqs. (6) and (7) to

$\rho_{k, \Lambda}^{A}(s ; m)=\frac{\Gamma\left(m, m s k^{2-\eta}\right)-\Gamma\left(m, m s \Lambda^{2-\eta}\right)}{\Gamma(m)}$,

and

$r_{\Lambda}^{A}=(2-\eta) \frac{\left(m s \Lambda^{2-\eta}\right)^{m}}{\Gamma(m)} e^{-m s \Lambda^{2-\eta}}$.

We will call this a type-A' regulator.

Expressing the functional trace in terms of a momentum integral, the PT flow equation reads

$\Lambda \frac{d}{d \Lambda} S_{\Lambda}[\phi]=\frac{1}{2} \int d^{d} x \int \frac{d^{d} p}{(2 \pi)^{d}}\left\langle x\left|r_{\Lambda} e^{-s S_{\Lambda}^{(2)}[\phi]}\right| p\right\rangle\langle p \mid x\rangle$.

This can be evaluated by employing the derivative expansion within the functional trace and then performing the momentum integral. We will take into account all terms up to two derivatives, i.e., we will calculate the flow of $Z_{\Lambda}(\phi)$ and $V_{\Lambda}(\phi)$. Thus, for evaluating the functional trace, we need to commute all derivatives to the right,

$\left[\partial_{\mu}, f(\phi)\right]=f^{\prime}(\phi)\left(\partial_{\mu} \phi\right)$

in order to make use of the identity $\partial_{\mu}|p\rangle=i p_{\mu}\left|p_{\mu}\right\rangle$ and finally project the flow on the running of $Z_{\Lambda}(\phi)$ and $V_{\Lambda}(\phi)$. The details of the derivation as well as the equations are provided in the appendix, where the flow equations for the dimensionless versions of $Z_{\Lambda}$ and $V_{\Lambda}$ (denoted $z$ and $v$ ) are given in Eqs. (61) and (62).

The fixed-point solutions are obtained by means of a multiple shooting method. In particular (see [8] for an extended discussion), a large field expansion is assumed to be valid at some fixed value of the field where the initial conditions are set, and an inward numerical integration of the fixed point Eqs. (61) and (62) is performed. At $x=0$ instead the initial condition for the outward integration is performed assuming $z(x=0)=1, z^{\prime}(x=0)=v^{\prime}(x=0)=0$. For

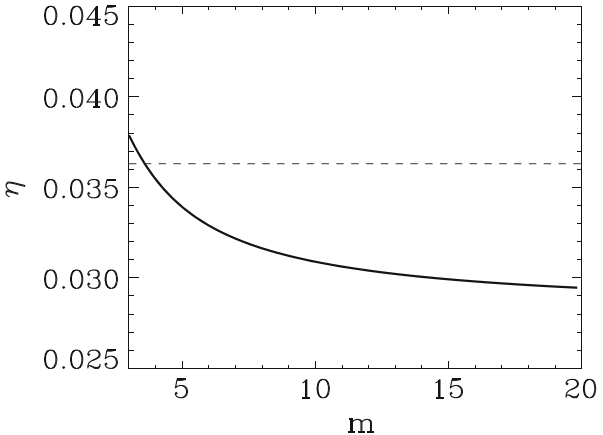

Fig. 1 The anomalous dimension $\eta$ for the regulator considered (typeA) in this work as a function of the proper-time parameter $m$. In the $m=$ $\infty$ limit we find $\eta=0.0294$. The dashed line indicates $\eta=0.03629$ obtained with conformal bootstrap method which is the most accurate determination nowadays

actual calculations the Runge-Kutta integrator DO2PVF ${ }^{2}$ turned out to be rather efficient. Continuity at the fitting point of the functions $Z, V$ and their derivatives is obtained by means of a globally convergent Broyden's method. ${ }^{3}$ In particular a tolerance of $10^{-6}$ for the root finding algorithm has been assumed. The result for the anomalous dimensions are depicted in Fig. 1.

The critical exponent $v$ is instead almost constant as a function of $m$ and its value is $v=0.613$.

Let us also mention the equations for the other types of coarse-graining schemes (type B and C) previously discussed. We shall derive the flow equations but postpone to another work the full numerical analysis for these schemes. We introduce a regulator, field dependent in general, of the form, cf. Eq. (6),

$$
\begin{aligned}
r_{\Lambda}^{p}(s ; m)= & \left(2-\eta(1-p)+\alpha p \Lambda \partial_{\Lambda} \ln Z_{\Lambda}(\phi)^{p}\right) \\
& \frac{\left(m s Z_{\Lambda}(\phi)^{p} \Lambda^{2-\eta(1-p)}\right)^{m}}{\Gamma(m)} e^{-m s Z_{\Lambda}(\phi)^{p} \Lambda^{2-\eta(1-p)}} .
\end{aligned}
$$

The various classes of regulators can be distinguished in terms of the parameters $p$ and $\alpha$. For $p=0$ we have a regulator of type- $A$ while for $p=1$ we have regulators of type $\mathrm{B}$ or $\mathrm{C}$ for $\alpha=1$ or $\alpha=0$, respectively.

In addition, in the type- $C$ regulator one can replace

$$
\begin{aligned}
& \int \mathrm{d}^{d} x r_{\Lambda}^{p}(\phi) f(\phi)(\square \phi) \\
& \rightarrow-\int \mathrm{d}^{d} x\left[r_{\Lambda}^{p}(\phi) f^{\prime}(\phi)+\beta r_{\Lambda}^{p^{\prime}}(\phi) f(\phi)\right]\left(\partial^{\mu} \phi\right)\left(\partial_{\mu} \phi\right) .
\end{aligned}
$$

\footnotetext{
${ }^{2}$ Implemented by the NAG group (see https://www.nag.com for details).

${ }^{3}$ This is described for example in http://numerical.recipes.
} 
In the full type- $C$ case one should keep $\beta=1$. Historically, the PT flow equations used in the literature have been derived heuristically by a simple RG-improvement of the 1-loop proper-time expression, [28-30] and that simplified type-C scheme corresponds to setting $\beta=0$. On the contrary, regulators of type $A$ and $B$ have never been used to calculate the critical exponents for the Wilson-Fisher fixed point.

The flow equations for $v$ and $z$ in these spectrally adjusted coarse-graining schemes are given in the Appendix in Eqs. (63) and (64) as a function of $p, \alpha$ and $\beta$.

\section{Conclusions}

We have argued that, at least at a formal level, the PTRG Eq. (2) and its generalization (3) can indeed be seen as Wilsonian RG equations. In fact, we found that each such flow can be associated to an infinite family of coarse-graining procedures. In practical applications, this freedom could be exploited to avoid certain pathologies.

The definition of the flow depends, as usual, on several choices. Foremost among these is the choice of a regulator function. Among all possible definitions, we have considered certain regulators based on incomplete Gamma functions. Then, we have considered the freedom of introducing a "spectral adjustment" either in the cutoff itself or in its derivative with respect to $\log \Lambda$. These spectral choices have the advantage of making possible a more direct interpretation for the coarse-graining as a suppression of the modes with $p^{2}>\Lambda^{2}$. We have implemented the flow equation of the Ising universality class, using a regulator without spectral adjustment (type-A). Depending on the value of the parameter $m$ the results for the anomalous dimension $\eta$ and for the exponent $v$ in the scheme-A are actually of a quality a bit lower compared to other RG equations. From a preliminary analysis they are improving moving to the spectrally adjusted $\mathrm{RG}$ equations (type B and C). Such results will be discussed elsewhere.

Acknowledgements We thank Tim Morris, Jan Pawlowski and Kevin Falls for interesting discussions. S.L. is supported by the DFG Collaborative Research Center "SFB 1225 (ISO-QUANT)".

Data Availability Statement This manuscript has no associated data or the data will not be deposited. [Authors' comment: This manuscript does not have associated data in a data repository.]

Open Access This article is licensed under a Creative Commons Attribution 4.0 International License, which permits use, sharing, adaptation, distribution and reproduction in any medium or format, as long as you give appropriate credit to the original author(s) and the source, provide a link to the Creative Commons licence, and indicate if changes were made. The images or other third party material in this article are included in the article's Creative Commons licence, unless indicated otherwise in a credit line to the material. If material is not included in the article's Creative Commons licence and your intended use is not permitted by statutory regulation or exceeds the permitted use, you will need to obtain permission directly from the copyright holder. To view a copy of this licence, visit http://creativecomm ons.org/licenses/by/4.0/.

Funded by $\mathrm{SCOAP}^{3}$.

\section{Appendix: Derivative expansion}

In this appendix the main steps to obtain the flow equation in the derivative expansion are given. As this involves quite some algebra, we use the Mathematica package xAct [31, 32]. However, in a first step we split the second functional derivative into two pieces,

$S_{\Lambda}^{(2)}[\phi]=S_{0}^{(2)}[\phi]+\delta S^{(2)}[\phi]$,

the first contains the box operator,

$S_{0}^{(2)}[\phi]=Z_{\Lambda}(\phi) \square$,

and the second is the remainder,

$$
\begin{aligned}
\delta S^{(2)}[\phi]= & -Z_{\Lambda}^{\prime}(\phi)\left(\partial^{\mu} \phi\right) \partial_{\mu}+Z_{\Lambda}^{\prime}(\phi)(\square \phi) \\
& -\frac{1}{2} Z_{\Lambda}^{\prime \prime}(\phi)\left(\partial_{\mu} \phi\right)\left(\partial^{\mu} \phi\right)+V_{\Lambda}^{\prime \prime}(\phi) .
\end{aligned}
$$

This split allows us to only take into account finitely many commutators when evaluating the exponential in Eq. (42), as higher orders lie outside of our truncation. Using the Zassenhaus (Baker-Campbell-Haussdorff) formula we find

$$
\begin{aligned}
e^{-s S_{\Lambda}^{(2)}[\phi]}= & e^{\frac{s^{3}}{3}\left[\delta S^{(2)}[\phi], S_{0}^{(2)}[\phi]\right]_{2}-\frac{s^{3}}{6}\left[S_{0}^{(2)}[\phi], \delta S^{(2)}[\phi]\right]_{2}} \\
& e^{\frac{s^{2}}{2}\left[S_{0}^{(2)}[\phi], \delta S^{(2)}[\phi]\right]} e^{-s \delta S^{(2)}[\phi]} e^{-s S_{0}^{(2)}[\phi]}+\mathcal{O}\left(\partial^{3} \phi\right),
\end{aligned}
$$

where $[X, Y]_{n+1}=\left[X,[X, Y]_{n}\right]$ and $[X, Y]_{0}=Y$. The first two exponentials can be expanded to finite order, as they contain at least one derivative of the field. For the third exponential, we perform a second split. We rewrite $\delta S^{(2)}[\phi]$ as,

$\delta S^{(2)}[\phi]=\delta S_{\partial}^{(2)}[\phi]+\delta S_{0}^{(2)}[\phi]$,

where $\delta S_{\partial}^{(2)}[\phi]$ contains an actual operator,

$\delta S_{\partial}^{(2)}[\phi]=-Z_{\Lambda}^{\prime}(\phi)\left(\partial^{\mu} \phi\right) \partial_{\mu}$,

while $\delta S_{0}^{(2)}[\phi]$ is just a number,

$\delta S_{0}^{(2)}[\phi]=Z_{\Lambda}^{\prime}(\phi)(\square \phi)-\frac{1}{2} Z_{\Lambda}^{\prime \prime}(\phi)\left(\partial_{\mu} \phi\right)\left(\partial^{\mu} \phi\right)$ 


$$
+V_{\Lambda}^{\prime \prime}(\phi)
$$

So we can use the Zassenhaus Formula again,

$$
\begin{aligned}
e^{-s \delta S^{(2)}[\phi]}= & e^{-\frac{s^{2}}{2}\left[\delta S_{\partial}^{(2)}[\phi], \delta S_{0}^{(2)}[\phi]\right]} e^{-s \delta S_{\partial}^{(2)}[\phi]} e^{-s \delta S_{0}^{(2)}[\phi]} \\
& +\mathcal{O}\left(\partial^{3} \phi\right),
\end{aligned}
$$

and as before, we can expand the first two exponentials to finite order. Finally, we need to evaluate the most right exponent in (49), $e^{-s S_{0}^{(2)}}$. For this let us decompose $\left(Z_{\Lambda}(\phi) \square\right)^{n}$,

$$
\left(Z_{\Lambda}(\phi) \square\right)^{n}=\sum_{i=0}^{4} a_{n}^{i} I_{i} Z_{\Lambda}(\phi)^{n-m_{i}} \square^{n-m_{i}}+\mathcal{O}\left(\partial^{3} \phi\right),
$$

in terms of the invariants $I_{i}$ containing up to two derivatives of the field,

$$
\begin{aligned}
I_{0} & =\mathbb{1}, \quad I_{1}=\left[\square, Z_{\Lambda}(\phi)\right], \quad I_{2}=\left[Z_{\Lambda}(\phi), \square\right]_{2} \square, \\
I_{3} & =\left[\square, Z_{\Lambda}(\phi)\right]^{2} Z_{\Lambda}(\phi) \square, \quad I_{4}=\left[\square, Z_{\Lambda}(\phi)\right]_{2} Z_{\Lambda}(\phi), \\
m_{0} & =0, \quad m_{1}=1, \quad m_{2}=2, \\
m_{3} & =3, \quad m_{4}=2 .
\end{aligned}
$$

By induction one can show that the $a_{n}^{i}$ satisfy the recursion relation,

$$
\begin{aligned}
& a_{n+1}^{0}=1, \quad a_{n+1}^{1}=a_{n}^{1}+n, \\
& a_{n+1}^{2}=-a_{n}^{1}+a_{n}^{2}-\frac{n(n+1)}{2}, \\
& a_{n+1}^{3}=(n-1) a_{n}^{1}+a_{n}^{3}, \\
& a_{n+1}^{4}=a_{n}^{1}+a_{n}^{4},
\end{aligned}
$$

with the initial conditions

$$
a_{0}^{0}=a_{1}^{0}=1, \quad a_{0}^{i>0}=a_{1}^{i>0}=0 .
$$

The solution of this recursion reads

$$
\begin{aligned}
& a_{n}^{0}=1, \quad a_{n}^{1}=\frac{n(n-1)}{2}, \\
& a_{n+1}^{2}=-\frac{n(n-1)(2 n-1)}{6}, \\
& a_{n+1}^{3}=\frac{n(n-1)(n-2)(3 n-5)}{24}, a_{n+1}^{4} \\
& =\frac{n(n-1)(n-2)}{6} .
\end{aligned}
$$

After determining the $a_{n}^{i}$ we can evaluate the exponential,

$e^{-s S_{0}^{(2)}}=\sum_{n=0}^{\infty} \frac{(-s)^{n}}{n !}\left(Z_{\Lambda}(\phi) \square\right)^{n}$

$$
\begin{aligned}
& =\sum_{n=0}^{\infty} \frac{(-s)^{n}}{n !} \sum_{i=0}^{4} a_{n}^{i} I_{i} Z_{\Lambda}(\phi)^{n-m_{i}} \square^{n-m_{i}}+\mathcal{O}\left(\partial^{3} \phi\right) \\
& =\sum_{i=0}^{4} I_{i} \sum_{n=0}^{\infty} \frac{(-s)^{n}}{n !} a_{n}^{i} Z_{\Lambda}(\phi)^{n-m_{i}} \square^{n-m_{i}}+\mathcal{O}\left(\partial^{3} \phi\right),
\end{aligned}
$$

by using the identity

$\sum_{n=0}^{\infty} \frac{n^{m}}{n !} x^{n}=\left(x \partial_{x}\right)^{m} e^{x}$.

At last, the dimensionless quantities $x=\phi \Lambda^{-\frac{d-2+\eta}{2}}$, $v=V \Lambda^{-d}$ and $z=Z \Lambda^{\eta}$ where $\eta$ is the anomalous dimension, are introduced. For a type- $A$ cutoff $(p=0)$ the flow equations for $v$ and $z$ read

$$
\begin{aligned}
\dot{v}= & d v-\frac{1}{2} x(d+\eta-2) v^{\prime} \\
& +\frac{2^{-d-1} \pi^{-d / 2}(\eta-2) m^{m} z^{-d / 2} \Gamma\left(m-\frac{d}{2}\right)\left(m+v^{\prime \prime}\right)^{\frac{1}{2}(d-2 m)}}{\Gamma(m)}
\end{aligned}
$$

$$
\begin{aligned}
\dot{z}= & -z \eta-\frac{1}{2} x(d+\eta-2) z^{\prime}-2^{-d-4} \pi^{-d / 2} \\
\times & (\eta-2) m^{m} z^{-\frac{d}{2}-1} \frac{\Gamma\left(1-\frac{d}{2}+m\right)}{3 \Gamma(m)} \\
& \left(\left(v^{(3)}\right)^{2} z^{2}(d-2(m+1))(d-2(m+2))\right. \\
& +\left(m+v^{\prime \prime}\right)^{2}\left(((d-18) d-4)\left(z^{\prime}\right)^{2}+24 z z^{\prime \prime}\right) \\
& \left.-2(d-10) v^{(3)} z(d-2(m+1)) z^{\prime}\left(m+v^{\prime \prime}\right)\right)
\end{aligned}
$$

For type-B and type C cutoff ( $p=1$ ) the flow equations for $v$ and $z$ are significantly more involved. Unified formulae read

$$
\begin{aligned}
\dot{v}= & d v-\frac{1}{2} x(d+\eta-2) v^{\prime}+\frac{\gamma}{z}\left(\frac{z}{m z+v^{\prime \prime}}\right)^{m-\frac{d}{2}} \\
& \times\left(\alpha x(d+\eta-2) z^{\prime}+2 z(\alpha \eta-2)+2 \alpha \dot{z}\right)
\end{aligned}
$$

where $\gamma=m^{m} \Gamma\left[m-\frac{d}{2}\right] / \Gamma[m] \pi^{d / 2} 2^{2+d}$. The flow equation for $z$ instead reads

$$
\begin{aligned}
\dot{z}= & \beta 2^{-d-4} \delta z^{-\frac{d}{2}+m-2}\left(m z+v^{\prime \prime}\right)^{\frac{1}{2}(d-2(m+3))} \\
& \times\left(z ^ { 2 } \left(\alpha\left(d^{2}-14 d+40\right) m^{2} x(d+\eta-2)\left(z^{\prime}\right)^{3}\right.\right. \\
& +2 \alpha m \dot{z} z^{\prime}\left(\left(d^{2}-14 d+40\right) m z^{\prime}\right. \\
& \left.-(d-6) v^{(3)}(d-2(m+1))\right)-2 \alpha v^{(3)} \\
\times & (d-2(m+1)) v^{\prime \prime}\left(x(d+\eta-2) z^{\prime \prime}+2 \dot{z}^{\prime}\right) \\
& +m\left(z^{\prime}\right)^{2}\left(2(d-10) v^{\prime \prime}(-4 \alpha+d(\alpha(\eta+2)-2)\right. \\
& +2 m(\alpha \eta-2)+4)-\alpha(d-6) v^{(3)} x(d+\eta-2) \\
\times & (d-2(m+1)))+2 v^{\prime \prime} z^{\prime}\left(v^{(3)}(-d\right. \\
& +2 m+2)(\alpha(d+\eta-2)
\end{aligned}
$$




$$
\begin{aligned}
& +2 m(\alpha \eta-2))+2 \alpha(d-10) m x(d+\eta-2) z^{\prime \prime} \\
& \left.\left.+4 \alpha(d-10) m \dot{z}^{\prime}\right)\right)+z v^{\prime \prime} z^{\prime}\left(2 \alpha \dot { z } \left(2 v^{(3)}(-d m+d\right.\right. \\
& \left.\left.+2 m^{2}-2\right)+(d-10) m(d+2 m-6) z^{\prime}\right) \\
& +2 z^{\prime}\left(\alpha(m-1) v^{(3)} x(d+\eta-2)(-d+2 m+2)\right. \\
& \left.+(d-10) v^{\prime \prime}(\alpha(d+\eta-2)+2 m(\alpha \eta-2))\right) \\
& +\alpha(d-10) m x(d+\eta-2)(d+2 m-6)\left(z^{\prime}\right)^{2} \\
& \left.+2 \alpha(d-10) v^{\prime \prime}\left(x(d+\eta-2) z^{\prime \prime}+2 \dot{z}^{\prime}\right)\right) \\
& +2 m z^{3}\left(-\alpha v^{(3)}(d-2(m+1))\right. \\
& \times\left(x(d+\eta-2) z^{\prime \prime}+2 \dot{z}^{\prime}\right) \\
& +z^{\prime}\left(-v^{(3)}(d-2(m+1))(-\alpha(3 \eta+2)\right. \\
& +d(\alpha \eta+\alpha-2)+8)+\alpha(d-10) m x(d+\eta-2) z^{\prime \prime} \\
& \left.+2 \alpha(d-10) m \dot{z}^{\prime}\right)+(d-10) m\left(z^{\prime}\right)^{2} \\
& \times(-\alpha(\eta+2)+d(\alpha \eta+\alpha-2)+4)) \\
& \left.+2 \alpha(d-10)(m-1)\left(v^{\prime \prime}\right)^{2}\left(z^{\prime}\right)^{2}\left(x(d+\eta-2) z^{\prime}+2 \dot{z}\right)\right) \\
& -2^{-d-5} \delta z^{-\frac{d}{2}+m-2}\left(m z+v^{\prime \prime}\right)^{\frac{1}{2}(d-2(m+3))}\left(z ^ { 2 } \left(\left(v^{(3)}\right)^{2}\right.\right. \\
& \times\left(d^{2}-2 d(2 m+3)+4\left(m^{2}+3 m+2\right)\right) \\
& +\left(d^{2}-18 d-4\right) m^{2}\left(z^{\prime}\right)^{2}-2(d-10) m v^{(3)} \\
& \left.\times(d-2(m+1)) z^{\prime}+48 m v^{\prime \prime} z^{\prime \prime}\right) \\
& \times+2 z v^{\prime \prime}\left(\left(d^{2}-18 d-4\right) m\left(z^{\prime}\right)^{2}-(d-10) v^{(3)}\right. \\
& \left.\times(d-2(m+1)) z^{\prime}+12 v^{\prime \prime} z^{\prime \prime}\right)+\left(d^{2}-18 d-4\right)\left(v^{\prime \prime}\right)^{2}\left(z^{\prime}\right)^{2} \\
& \left.+24 m^{2} z^{3} z^{\prime \prime}\right)\left(\alpha x(d+\eta-2) z^{\prime}+2 z(\alpha \eta-2)+2 \alpha \dot{z}\right) \\
& -\frac{1}{2} x(d+\eta-2) z^{\prime}-\eta z
\end{aligned}
$$

where $\gamma=m^{m} \Gamma\left[m-\frac{d}{2}\right] / \Gamma[m] \pi^{d / 2} 2^{2+d}$ and $\delta=m^{m} \Gamma[1-$ $d / 2+m] / \Gamma[m] 3 \pi^{d / 2}$. Setting $\alpha=\beta=1, \alpha=0$ and $\beta=1$, $\alpha=\beta=0$ one obtains schemes $B, C$ and simplified-C respectively, as discussed in the end of Sect. 4.

\section{References}

1. K.G. Wilson, Rev. Mod. Phys. 47, 773 (1975)

2. F.J. Wegner, A. Houghton, Renormalization group equation for critical phenomena. Phys. Rev. A 8, 401 (1973)

3. J. Polchinski, Nucl. Phys. B 231, 269 (1984)

4. C. Wetterich, Average action and the renormalization group equations. Nucl. Phys. B 352, 529 (1991)

5. C. Wetterich, Exact evolution equation for the effective potential. Phys. Lett. B 301, 90 (1993)

6. T.R. Morris, Int. J. Mod. Phys. A 9, 2411 (1994). arXiv:hep-ph/9308265

7. T.R. Morris, Derivative expansion of the exact renormalization group. Phys. Lett. B 329, 241 (1994). arXiv:hep-ph/9403340

8. T.R. Morris, On truncations of the exact renormalization group. Phys. Lett. B 334, 355 (1994). arXiv:hep-th/9405190
9. T.R. Morris, Prog. Theor. Phys. Suppl. 131, 395-414 (1998). arXiv:hep-th/9802039

10. C. Bagnuls, C. Bervillier, Exact renormalization group equations: an introductory review. Phys. Rep. 348, 91 (2001). arXiv:hep-th/0002034

11. J. Berges, N. Tetradis, C. Wetterich, Non-perturbative renormalization flow in quantum field theory and statistical physics. Phys. Rep. 363, 223 (2002). arXiv:hep-ph/0005122

12. B. Delamotte, An introduction to the nonperturbative renormalization group, Lect. Notes Phys. 852 (2012) 49-132, arXiv:cond-mat/0702365

13. O.J. Rosten, Fundamentals of the exact renormalization group. Phys. Rep. 511, 177 (2012). arXiv:1003.1366 [hep-th]

14. M. Reuter, C. Wetterich, Effective average action for gauge theories and exact evolution equations. Nucl. Phys. B 417, 181 (1994)

15. H. Gies, Running coupling in Yang-Mills theory: a flow equation study. Phys. Rev. D 66, 025006 (2002). arXiv:hep-th/0202207

16. J.M. Pawlowski, Aspects of the functional renormalization group. Ann. Phys. 322, 2831-2915 (2007). arXiv:hep-th/0512261

17. R. Floreanini, R. Percacci, The Heat kernel and the average effective potential. Phys. Lett. B 356, 20 (1995). arXiv:hep-th/9505172

18. S.B. Liao, On connection between momentum cutoff and the proper time regularization. Phys. Rev. D 53, 2020 (1996). arXiv:hep-th/9501124

19. D.F. Litim, J.M. Pawlowski, Predictive power of renormalization group flows: a comparison. Phys. Lett. B 516, 197 (2001). arXiv:hep-th/0107020

20. D.F. Litim, J.M. Pawlowski, Perturbation theory and renormalization group equations. Phys. Rev. D 65, 081701 (2002). arXiv:hep-th/0111191

21. D.F. Litim, J.M. Pawlowski, Completeness and consistency of renormalisation group flows. Phys. Rev. D 66, 025030 (2002). arXiv:hep-th/0202188

22. D. Zappala, Perturbative and nonperturbative aspects of the proper time renormalization group. Phys. Rev. D 66, 105020 (2002). arXiv:hep-th/0202167

23. A. Bonanno, G. Lacagnina, Spontaneous symmetry breaking and proper time flow equations. Nucl. Phys. B 693, 36 (2004). arXiv:hep-th/0403176

24. A. Bonanno, M. Reuter, Proper time flow equation for gravity. JHEP 0502, 035 (2005). https://doi.org/10.1088/1126-6708/2005/ 02/035. arXiv:hep-th/0410191

25. S.P. de Alwis, Exact RG flow equations and quantum gravity. JHEP 1803, 118 (2018). arXiv:1707.09298 [hep-th]

26. D.F. Litim, J.M. Pawlowski, Wilsonian flows and background fields. Phys. Lett. B 546, 279 (2002). arXiv:hep-th/0208216

27. J.I. Latorre, T.R. Morris, Exact scheme independence. JHEP 0011, 004 (2000). arXiv:hep-th/0008123

28. D.F. Litim, D. Zappala, Ising exponents from the functional renormalisation group. Phys. Rev. D 83, 085009 (2011). arXiv:1009.1948 [hep-th]

29. M. Mazza, D. Zappala, Proper time regulator and renormalization group flow. Phys. Rev. D 64, 105013 (2001). arXiv:hep-th/0106230

30. A. Bonanno, D. Zappala, Towards an accurate determination of the critical exponents with the renormalization group flow equations. Phys. Lett. B 504, 181 (2001). arXiv:hep-th/0010095

31. J.M. Martín-García, D. Yllanes, R. Portugal, The Invar tensor package: differential invariants of Riemann. Comput. Phys. Commun. 179, 586-590 (2008). arXiv:0802.1274 [cs.SC]

32. xAct: Efficient tensor computer algebra for Mathematica, http:// xact.es/index.html. Online; accessed 16-December-2019 